\title{
Análise Interativa Qualitativa: uma contribuição metodológica para pesquisas em sustentabilidade
}

\section{Interactive Qualitative Analysis: a methodological contribution to researches in sustainability}

\author{
Silvia Ferraz Nogueira de Tommaso \\ Ivete Rodrigues \\ Vanessa Cuzziol Pinsky
}

Este estudo discute a utilização do método Interactive Qualitative Analysis (IQA) para operacionalização de uma pesquisa que investigou a adoção de estratégias de valor compartilhado em empresas de grande porte. Os estudos em sustentabilidade lidam com problemas complexos que requerem abordagens metodológicas interdisciplinares e sistêmicas. O método IQA oferece uma abordagem sistêmica e estruturada para a coleta e análise de dados em pesquisas qualitativas. Tanto quanto possível, busca-se diminuir os vieses do pesquisador, posto que os constituintes do estudo são os responsáveis por interpretar os dados, enquanto é reservado ao pesquisador um papel de facilitador do processo de análise. O que diferencia o IQA de outras formas de investigação qualitativa é que ele fornece um roteiro com procedimentos metodológicos transparentes e rastreáveis. A sistematização dos protocolos, o rigor metodológico, e as múltiplas fontes de coleta e análise de dados sugerem que o IQA é um método adequado para estudos na temática da sustentabilidade, com potencial de contribuir para uma literatura ainda em construção. $\mathrm{O}$ artigo agrega valor à prática acadêmica pois, mediante uma abordagem inovadora, oferece uma discussão aprofundada sobre a utilização do IQA em pesquisas relacionadas à sustentabilidade $A$ rastreabilidade metodológica do IQA permite que outros pesquisadores possam investigar o mesmo fenômeno em contextos diferentes ou, ainda, em um mesmo contexto envolvendo outros constituintes.

Palavras chaves: Método IQA; Sustentabilidade; Valor Compartilhado; Estratégia

This study discusses the use of the Interactive Qualitative Analysis Method (IQA) to operationalize a research that investigated the adoption of shared value strategies in large companies. Sustainability studies deal with complex problems that require interdisciplinary and systemic methodological approaches. The IQA method offers a structured and systemic approach to col-
Recebido em: 13/04/2021

Aprovado em: 24/06/2021

Silvia Ferraz Nogueira de Tommaso iD
silviatommaso@usp.br
Mestre em gestão de negócios - Faculdade
FIA de Administração e Negócios
MSc in Business Managment - Faculdade
FIA de Administração e Negócios
São Paulo / SP - Brasil
Ivete Rodrigues iD
iveter@fia.com.br
Doutora em Administração de Empresas -
Faculdade de Economia, Administração e
Contabilidade da USP
PhD in Business Administration - Faculdade
de Economia, Administração e Contabilidade
da USP
São Paulo / SP - Brasil
Vanessa Cuzziol Pinsky (D)
vanessa.pinsky@usp.br
Doutora em Administração de Empresas -
Faculdade de Economia, Administração e
Contabilidade da USP
PhD in Business Administration - Faculdade
de Economia, Administração e Contabilidade
da USP
São Paulo / SP - Brasil


lecting and analyzing data in qualitative research. The aim is to reduce, as far as possible, the researcher's biases, since the constituents, and not the researcher as expert, do the analysis and interpretation of data. The researcher has the role of facilitating the analysis process. What differentiates the IQA from other forms of qualitative inquiry is that it provides a roadmap with transparent and traceable methodological procedures. The systematization of the protocols, the methodological rigor, and the multiple sources of data collection and analysis suggest that the IQA is an appropriate method for studies on the theme of sustainability, with the potential to contribute to a literature still under construction. The article adds value to academic practice because, through an innovative approach, it offers an in-depth discussion on the use of the IQA in research related to sustainability. The methodological traceability of the IQA allows other researchers to investigate the same phenomenon in different contexts or even in the same context involving other constituents.

Keywords: IQA Method, Sustainability, Shared Value; Strategy

\section{Introdução}

O tema da sustentabilidade está inserido num contexto de interações complexas entre diversas partes interessadas. Este fato ressalta a necessidade de integração de saberes e conhecimentos numa perspectiva interdisciplinar. Superando assim, paradigmas epistemológicos que analisam o mundo como algo homogêneo. O campo de pesquisa em sustentabilidade demanda abordagens metodológicas que considerem os desafios sociais, ambientais, econômicos, políticos, éticos e culturais, visando ao entendimento das relações intrínsecas entre o ser humano, a natureza e a sociedade (SANTOS; BASSI, 2017).

Nesse sentido, a complexidade dessa temática demanda novas técnicas de construção e análise de dados. Metodologias inovadoras e pensadores do século XX e XXI tem proposto outras maneiras de investigação científica com aplicações, principalmente, no campo das ciências sociais que se contrapõem ao positivismo, que apesar de ter trazido inúmeras descobertas científicas, tem sido questionado por não atender as demandas da sociedade atual (NIELSEN et al. ,2018).

Um dos teóricos que contestou o positivismo foi Kuhn (2013) ao estabelecer o conceito de paradigma científico, definido como um conjunto de crenças, valores e técnicas compartilhadas por membros de determinada comunidade científica. 
Para o teórico, a objetividade do positivismo não é possível, pois dois cientistas, por mais que compartilhem compromissos paradigmáticos, poderão chegar a conclusões diferentes a respeito de uma mesma teoria. As razões para isso são subjetivas, posto que relacionadas a questões profissionais, pessoais, ideológicas ou religiosas. Kuhn propõe que as análises teóricas não são determinadas por regras, mas sim condicionadas ou influenciadas por valores. Tais divergências, em vez de imperfeições a serem removidas, são uma parte essencial da natureza da ciência.

Corroborando com essa ideia, Morin (2011) argumenta que a natureza se compõe em sistemas abertos que se organizam a partir da interação com outros elementos. No lugar da especialização, da simplificação e da fragmentação de saberes, o estudioso propõe o conceito da complexidade. É complexo o que não pode se resumir numa palavra-chave, o que não pode ser reduzido a uma lei nem a uma ideia simples. A transdisciplinaridade propõe a quebra do paradigma do estudo das disciplinas de forma isolada, salientando que a incerteza e as contradições são como parte da vida e da condição humana. Ao mesmo tempo, sugere a solidariedade e a ética como caminho para a religação dos seres e dos saberes (MORIN, 2011), o que contrapõe à teoria positivista que estabelece que a máxima do conhecimento humano teria como missão descobrir e compilar as leis da natureza, as quais seriam precisas e invariáveis (NIELSEN et al., 2018).

Desta forma, os desdobramentos teóricos de Kuhn (2013) e a teoria da complexidade de Morin (2011) mostram-se como esteios epistemológicos para pesquisas no âmbito das ciências sociais. São pertinentes também à área de sustentabilidade, pois é um campo de estudo permeado de temas complexos que podem envolver de forma sistêmica as dimensões econômica, ambiental, social e de governança. Olhando a questão sob o ponto de vista dos métodos mais utilizados em pesquisas nesta seara, verifica-se que há uma predominância da abordagem qualitativa. Em pesquisa bibliométrica realizada por Souza e Ribeiro (2013) foi identificado que a produção brasileira sobre o tema, em periódicos nacionais (Qualis A1 a B2, no período de 1992 a 2011), está centrada em estudos qualitativos, com predominância de pesquisa bibliográfica, seguida do estudo de caso.

Outra pesquisa com a mesma metodologia, porém mais recente (2013-2017), realizada junto à base Scielo, reafirma o predomínio de estudos qualitativos com objetivos exploratórios, tendo a entrevista como principal instrumento de coleta de 
dados (SANTOS; DOS SANTOS; MOURA, 2017). O que muitas vezes, pode apresentar inúmeros desafios e comprometer os resultados dependendo da preparação do pesquisador e da pré-disposição do entrevistado em compartilhar conhecimento e valores por meio da sua percepção (BISHWAKARMA, 2017).

Por outro lado, pesquisas de abordagem qualitativa por vezes se utilizam de grupo focal para coleta de dados o que propicia a escuta de diferentes pontos de vista sobre um tema. Grupo focal é uma técnica onde um grupo de pessoas é entrevistado informalmente e questões sobre assuntos específicos são discutidas para explorar suas percepções, opiniões, crenças e atitudes. A junção de técnicas de entrevistas e grupo focal para coleta de dados pode trazer significativas contribuições para a pesquisa de abordagem qualitativa especialmente focada em entender a percepção de um determinado tipo de constituinte, como por exemplo de crianças e adolescentes ou participantes com formação multidisciplinar (BISHWAKARMA, 2017).

Corroborando com a ideia de utilizar-se de outras técnicas de coleta e análise de dados, Zappellini e Feuerschütte (2015) ressaltam o uso da triangulação metodológica para aumentar a confiabilidade dos resultados da pesquisa e suas conclusões. Os autores baseiam-se na definição de Denzin (2005), onde a triangulação se refere à produção de conhecimento e não ao resultado. A técnica permite que se tenha um processo de pesquisa mais completo.

Tendo em vista a importância de abordagens metodológicas sistêmicas, bem como a necessidade de diversificar os métodos de pesquisa utilizados em sustentabilidade, é importante buscar autores do campo de metodologia científica que tenham afinidade com os pressupostos epistemológicos apresentados. A proposição desse estudo é de que o método Interactive Qualitative Analysis (IQA), proposto por Northcutt e McCoy (2004), traz contribuições relevantes à pesquisa qualitativa por meio de protocolos baseados em sistemas, múltiplas fontes de coleta e análise de dados e uma participação ativa dos sujeitos (constituintes), que não só contribuem com seus depoimentos, mas também são envolvidos em rodadas de análise e validação dos dados empíricos.

O método usa o desenho de pesquisa qualitativa e técnica de análise que combinam a fenomenologia com a teoria dos sistemas (SANCHEZ, 2007). O IQA foi desenvolvido inicialmente para alunos de graduação, e se tornou referência em estu- 
dos de problemas sociais complexos onde os pesquisadores buscam compreender em profundidade o fenômeno por meio da coleta, codificação e análise de dados. A construção de respostas é feita juntamente com os participantes da pesquisa para que a percepção dos envolvidos torne-se ponto forte na análise dos resultados.

Embora a abordagem metodológica IQA tenha sido utilizada em pesquisas de diversas áreas, como em saúde, tecnologia e fortemente em estudos na área de educação (PUGALIA, 2016; BARGATE, 2014), em administração, especificamente na área de sustentabilidade, ainda é pouco utilizada. Nesse sentido, o método apresenta um significativo potencial de contribuição para a investigação de problemas de pesquisa complexos, baseados nos desafios do desenvolvimento sustentável e cuja percepção dos diferentes stakeholders é fundamental para o delineamento de possíveis caminhos na proposição de soluções.

O objetivo do estudo é descrever os protocolos e procedimentos operacionais do método IQA aplicados em uma pesquisa empírica na área de sustentabilidade. $O$ artigo apresenta resultados da utilização da metodologia em uma pesquisa que buscou compreender a adoção da Criação de Valor Compartilhado como estratégia de vantagem competitiva para conciliar rentabilidade e bem-estar social por empresas brasileiras de grande porte. Porém, o foco do artigo está no método utilizado e não nos resultados empíricos e substantivos da pesquisa.

O artigo está estruturado em quatro partes, além dessa introdução. Na segunda parte é feita uma explicação dos principais elementos que compõem o método IQA. A discussão dos resultados é apresentada na terceira parte. Por último, são apresentadas as contribuições que o método IQA pode trazer para estudos de fenômenos complexos na área de sustentabilidade e recomendações para estudos futuros.

\section{Análise Interativa Qualitativa (IQA)}

A Análise Qualitativa Interativa (em inglês Interactive Qualitative Analysis IQA) é um método de pesquisa desenvolvido por Northcutt e McCoy (2004) que combina dois objetivos centrais: investigar os elementos que compõe um sistema e suas relações e traduzir essa teoria na prática de pesquisa. Trata-se de uma abordagem de pesquisa qualitativa que busca fornecer uma estrutura sistêmica, rigoro- 
sa e confiável, adequada a estudos nos quais os pesquisadores desejam examinar como os fenômenos são socialmente construídos (NORTHCUTT; MCCOY, 2004).

As técnicas de coleta e análise de dados do método originaram-se de procedimentos de Gestão da Qualidade Total (TQM) que busca conhecimentos da força produtiva para resolver problemas e melhorar os processos industriais. Uma das premissas do IQA é que as pessoas mais próximas ao fenômeno são as mais apropriadas para interpretar os elementos do sistema e suas relações. Nos estágios iniciais da análise, as vozes dos constituintes(termo utilizado para designar os participantes) são privilegiadas sobre a do pesquisador. Os pressupostos do método abordam diretamente a dependência de conhecimento e as posições de poder entre o pesquisador e os participantes. Os constituintes são definidos como um grupo de pessoas que têm uma compreensão compartilhada do fenômeno (NORTHCUTT; MCCOY, 2004). Nessa pesquisa, eles foram identificados nos relatórios de sustentabilidades das empresas investigadas, a partir das funções exercidas e que estavam diretamente ligadas a implementação das estratégias do Valor Compartilhado. As funções são: CEO, diretor, gerente e analista de sustentabilidade. Dentre as sete empresas investigadas, a seleção dos constituintes se deu pela disponibilidade em participar da pesquisa de forma interativa.

A partir da definição dos constituintes, o método IQA pressupõe a formação do grupo focal. Uma das técnicas de amostragem para escolha do grupo focal é a amostragem por conveniência, desde que os critérios citados para a seleção dos constituintes sejam atendidos. Segundo Northcutt e McCoy (2004), um grupo focal deve ser composto de 8 e 12 participantes, e consiste em um trabalho coletivo com o propósito fundamental de ouvir e aprender com os membros do grupo. O objetivo é melhorar a compreensão geral de um constructo, questão ou fenômeno. $O$ pesquisador ouve os participantes e aprende com eles. Embora cada participante possa ter suas próprias ideias, um conjunto completamente novo de dados pode surgir quando os participantes interagem entre si.

O pesquisador atua como facilitador do grupo focal por meio de três processos relacionados à análise de conteúdo de Glaser e Strauss (1967): a) Codificação aberta (levantamento em que os participantes são livres para expressar percepções e experiências; b) Codificação indutiva (organização das percepções e experiências em grupos de categorias; e c) Codificação axial (nomeação dos grupos ou afinida- 
des). A codificação é o procedimento em que os dados são divididos, conceitualizados e se estabelece suas relações. É um processo analítico cujos objetivos são: construir a teoria, dar ao processo científico o rigor metodológico necessário, auxiliar o pesquisador a detectar os vieses, desenvolver o fundamento, a densidade, a sensibilidade e a integração necessária para gerar uma teoria (STRAUSS; CORBIN, 1990).

$\mathrm{Na}$ pesquisa qualitativa tradicional o pesquisador gera os temas, coleta os dados e faz as análises. No método IQA o observador e o observado são dependentes (ou interdependentes). Sendo assim, os constituintes são responsáveis pela geração de temas ou afinidades (BARGATE, 2014). Dessa forma, o método desafia os pressupostos tradicionais da pesquisa qualitativa que sugerem que o papel dos participantes é fornecer dados e que apenas o pesquisador é qualificado para analisá-los. Com o IQA, os constituintes geram, teorizam e interpretam seus próprios dados enquanto o pesquisador facilita o processo. A intenção é de que os vieses do pesquisador sejam minimizados no processo.

Do ponto de vista epistemológico, o IQA considera que as pessoas conhecem seu mundo por meio da construção social de significados. Tanto a dedução quanto a indução são consideradas necessárias para a investigação dos significados e suas relações de influência. Os autores tomam emprestada a metáfora do bricoleur (artesão) de Denzin e Lincoln (2000) ao propor que o IQA oferece aos participantes a chance de "criar" significados ou representações de sistemas através de "colchas de retalho".

Quatro pontos são essenciais na estrutura do método IQA. O primeiro refere-se ao desenho da pesquisa, que deve ilustrar o processo heurístico de concepção de um estudo qualitativo interativo. O segundo aspecto importante é a realidade de grupo e os elementos do sistema que introduzem a codificação usada para identificar, esclarecer e descrever afinidades. O terceiro ponto é uso de um protocolo formal, mediante o qual os participantes constroem sistematicamente hipóteses fundamentadas nos dados para determinar a existência e a direcionalidade das influências existentes. O objetivo é resumir a estrutura subjacente em um mapa mental das percepções do grupo nas relações de causa e efeito entre todas as afinidades em um sistema. E por fim, a realidade individual é de grande importância, captada por meio de entrevistas individuais que validam a percepção coletiva desenvolvida no grupo focal. 
O desenho de pesquisa é um elemento chave no desdobramento e sucesso do método IQA. Corroborando com essa ideia, Lukosevicius (2018) aponta a importância do design da pesquisa. Segundo o autor, a inadequação e falta de maturação dos projetos de pesquisa são alguns dos problemas que mais levam à rejeição direta de artigos na área de administração.

Outro pressuposto fundamental de uma pesquisa é a definição do constructo, conceituado por Gil (2009) como uma construção mental criada a partir de elementos mais simples para representar o significado teórico de um conceito ou proposição. Um constructo apresenta validade quando reflete o seu verdadeiro significado. Para ser operacionalizado, o constructo é desdobrado em variáveis que, por sua vez, são características, propriedades ou aspectos observáveis. Com base nesses elementos, o pesquisador desenvolve hipóteses para serem averiguadas ao longo de sua pesquisa. Na linguagem do IQA, uma afinidade aproxima-se do conceito de variável da pesquisa qualitativa por ser reflexões sobre um constructo. Entretanto, elas se diferem na forma de medição. As afinidades não precisam ser operacionalizadas. Diferentemente das variáveis, elas são construídas dos pensamentos e palavras daqueles que detém conhecimento e influência sobre o fenômeno estudado. Tendem a ser mais ricas em significado e assumem uma unidade dialética dentro de um sistema. Para elucidar, crescimento (intelectual) como variável seria mensurada numa extensão entre confusão e clareza. Já como afinidade, ele seria uma representação dialética que sugere que o crescimento ocorre quando há interação constante e dinâmica ou tensão entre os dois polos opostos, confusão e clareza. Criando, assim, uma unidade de análise (NORTHCUTT; MCCOY, 2004, p. 83).

A Figura 1 apresenta o modelo teórico da pesquisa que é apresentada nesse artigo, descrevendo a relação entre constructos.

O primeiro constructo é a adoção da Criação de Valor Compartilhado (CVC). Trata-se de uma estratégia proposta por Porter e Kramer (2011), onde a empresa gera valor social ao mesmo tempo em que obtém retorno econômico. Os autores sugerem três formas de implementação da estratégia: a remodelação de produtos e mercados, a redefinição da produtividade da cadeia de valor e o desenvolvimento de cluster (arranjos produtivos locais). O segundo constructo refere-se à vantagem competitiva, definida por Barney (1991) como a criação de valor pela empresa de tal forma que suas atividades e capacidades as diferenciem de seus concorrentes. 
Esse constructo é medido pelo faturamento, receita, custos e fatia de mercado. $\mathrm{O}$ terceiro constructo é rentabilidade, definido pelo retorno financeiro sobre o valor investido, e operacionalizado pelas variáveis custo de produção, marketing ,vendas, estrutura fiscal e de negócio (SEBRAE, 2020). O quarto constructo é bem-estar social, cujas variáveis são métricas de educação, saúde, alimentação, moradia, trabalho e renda (IBGE, 2020).

Figura 1 Modelo Teórico

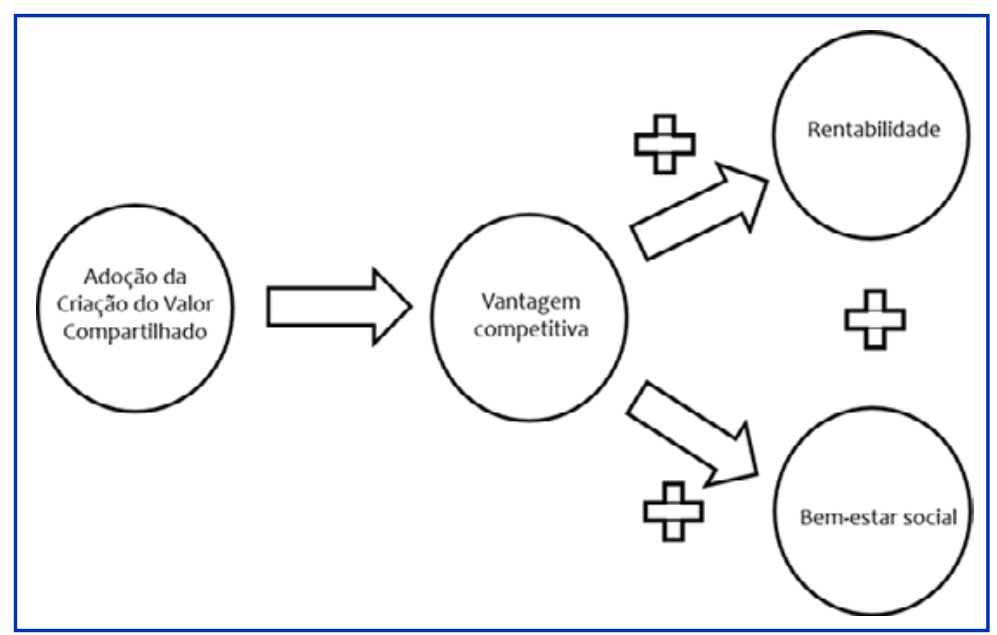

Fonte: Elaborado pelos autores (2021).

Com base nestes constructos e em suas variáveis a proposição estabelecida pelas pesquisadoras para interlocução com os constituintes foi: A adoção do valor compartilhado concilia a rentabilidade da empresa e o bem-estar da sociedade. A partir dessa sentença o trabalho do grupo focal se inicia. É no grupo focal que os constituintes emergem as afinidades a partir da proposição sugerida (Em função da pandemia do COVID 19, a pesquisa que ilustra esse artigo não pode desenvolver o grupo focal e a interação entre observados e observador foi feita por vido conferencia). Nesse sentido, o IQA é consistente com os princípios do construtivismo social pois "privilegia o significado socialmente construído" (NORTHCUTT; MCCOY, 2004, p. 4). O processo de pesquisa identifica as afinidades, isto é, os elementos de um fenômeno e suas relações compondo um sistema. E, por fim, as descrições de sig- 
nificação de cada relação. A representação visual é um mapa mental construído de forma coletiva pelos constituintes e pelo pesquisador. Este configura-se no Diagrama de Influência do Sistema (SID) que é o resultado final da pesquisa, elaborado de acordo com regras rigorosas e replicáveis com o objetivo de alcançar abrangência e interpretabilidade conforme afirmam Northcutt e McCoy (2004, p.41).

A Figura 2, etapas do IQA, apresenta um fluxograma típico do método IQA com as etapas da pesquisa e seu fluxo de operacionalização. As caixas do lado esquerdo retratam o trabalho com os constituintes do grupo focal até a formação do SID (Diagrama de Influências do Sistema). Já as caixas da direita retratam o fluxo das etapas de entrevista, culminando também no SID individual ou SID Composto, que consolida o resultado de mais de um entrevistado.

Figura 2 Etapas do IQA

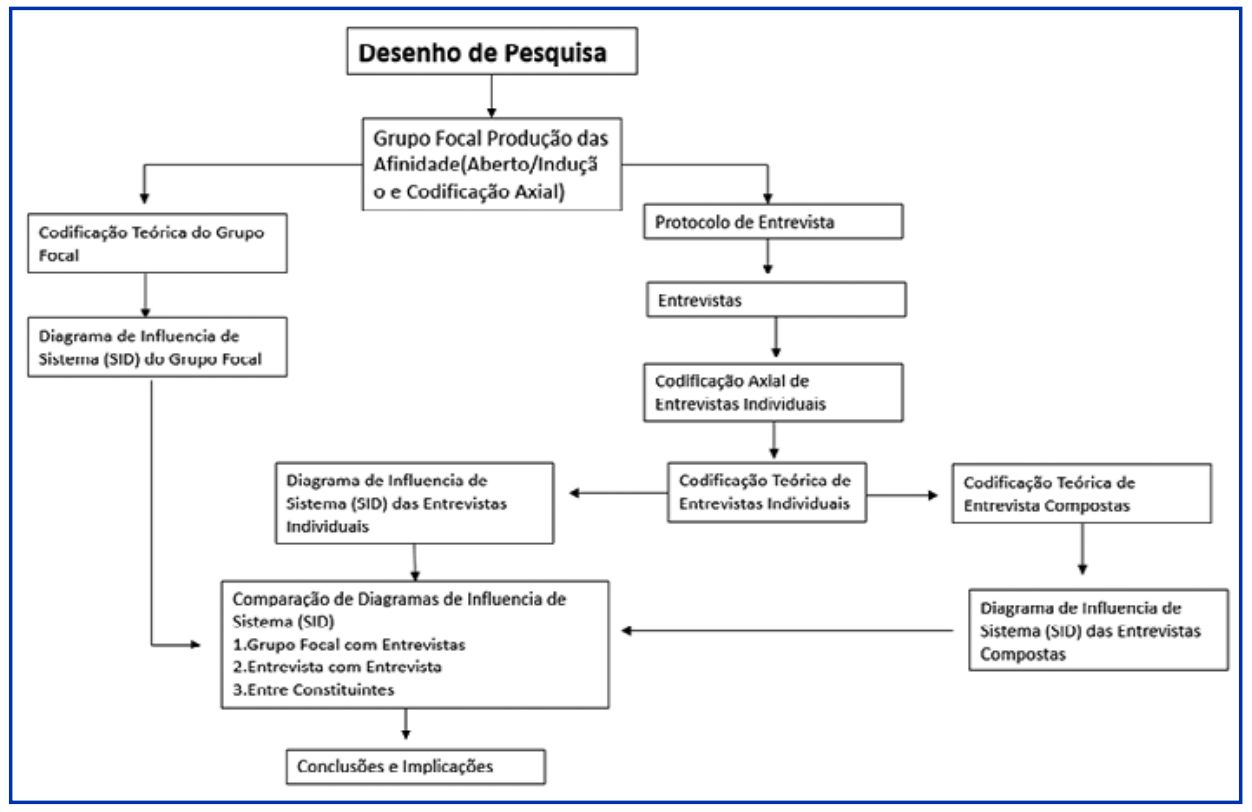

Fonte: Elaborado com base em NORTHCUTT, MCCOY (2004, p. 45).

Northcut e Mccoy (2004), descrevem o desenvolvimento do trabalho do grupo focal e a participação do pesquisador como um facilitador desse processo. A sessão se inicia com um brain storming (debate) onde os constituintes exploram 
as diferentes percepções sobre a proposição colocada pelo pesquisador. A partir desse debate, a etapa seguinte é a da codificação aberta, onde os participantes registram silenciosamente suas percepções em cartões de notas (post its). Após o registro os post-its são afixados em uma parede limpa e lidos em voz alta pelos constituintes. O facilitador (o pesquisador) lê cada post-it em voz alta mais uma vez, para garantir que os participantes entendam o que está escrito e para esclarecer algum ponto, se necessário.

Na sequência, é feita a codificação indutiva, momento em que os constituintes organizam os post-its em conjuntos com significados comuns. Isso continua até o consenso ser atingido, com a colocação de post-its em agrupamentos de afinidade produzindo uma visão coletiva. Novamente, este processo deve ser conduzido de forma individual e silenciosamente para evitar que indivíduos dominantes ou o facilitador monopolizem o processo. Na etapa final, por meio de codificação axial, os participantes exploram a relação entre os agrupamentos.

No IQA, há três possíveis relações de causa e efeito entre um par de afinidades que são nomeadas e posteriormente relacionadas. $A$ pode influenciar $B$, ou $B$ pode influenciar $A$ ou pode não haver relação entre $A$ e $B$. Os constituintes avaliam estas possíveis relações entre todas as afinidades a partir dos protocolos disponibilizados pelo método IQA. As afinidades definidas na pesquisa que ilustra esse artigo são: liderança com propósito, matriz de materialidade, ecossistema, desenvolvimento econômico sustentável, impacto, rentabilidade, bem-estar social, resultados de negócios e resultados sociais. O IQA sugere mais de duas e menos de 12 afinidades pela relevância de quantidade de relações. Nessa pesquisa pela análise feita através de codificação no Atlas.ti, nove afinidades foram determinadas pela frequência e relevância de citações.

As afinidades foram dispostas em sequência numérica por ordem alfabética sem nenhuma inferência de grau de importância. Todas as afinidades são igualmente importantes segundo o método. A sequência de atividades sugere o preenchimento da Tabela 1, ART (tabela de relação entre afinidades) pelos participantes. Essa tabela estabelece uma lista de possíveis relações entre um par de afinidades. Cada constituinte desenvolve uma tabela estabelecendo as relações entre as afinidades a partir de suas percepções e conhecimento. A Tabela é construída inserindo-se setas direcionadas de $A$ para $B(A \rightarrow B)$, o que indica que $A$ é a causa ou 
a afinidade de influência e $B$ é o efeito ou a afinidade influenciada, ou com a seta direcionada de $B$ para A seguindo o mesmo raciocínio de relação de causa e efeito. A terceira opção é deixar o espaço em branco indicando que não há relação entre $A$ e B. E os participantes descrevem a significação das relações sugeridas conforme exemplificado na Tabela 1, Relações entre afinidades.

Tabela 1 Relações entre Afinidades (ART)

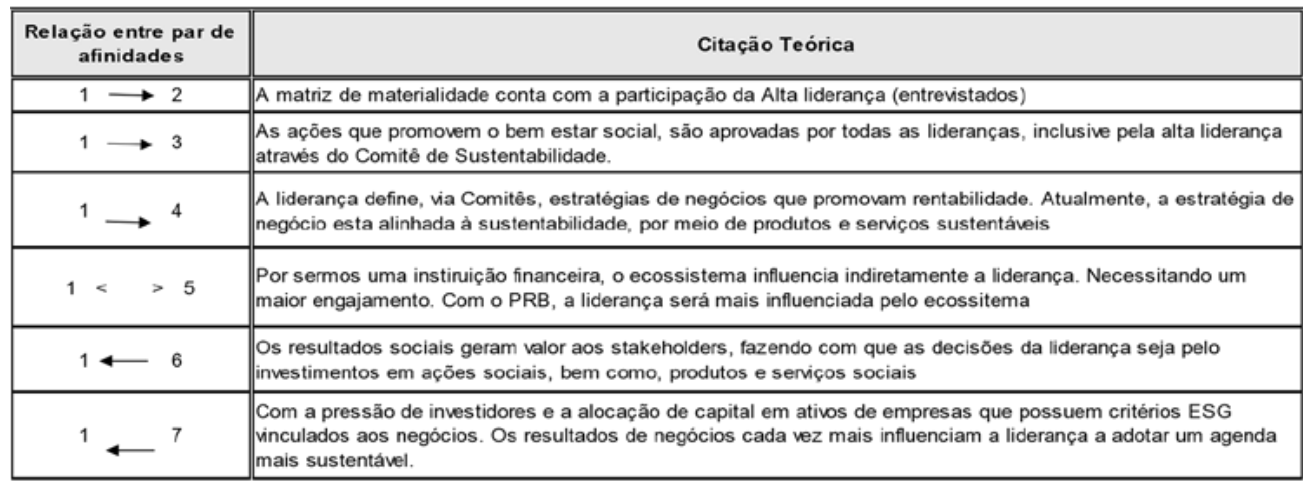

Fonte: Elaborado com base em NORTHCUTT, MCCOY (2004, p.153)

A partir disto, foram desenvolvidos os Diagramas de Inter-relação de Afinidades (IRD) que é o primeiro passo no processo de racionalizar o sistema. A Tabela 2, IRD mostra se a relação entre um par de afinidades pode ser uma causa percebida ou um efeito. Há também a possibilidade de ausência de relação entre o par de afinidades. As setas são posicionadas para cima $(\uparrow)$ OUTS ou para a esquerda $(\leftarrow)$ INS e cada relação é documentada duas vezes como num livro de contabilidade de entradas e saídas. Se uma relação foi determinada entre 1 e 2 e transcrita como $1 \leftarrow 2$, ela será lida como 2 influencia 1. E duas setas serão colocadas na Tabela para representar essa relação. Após o registro de todas as relações, o valor do Delta $(\Delta)$ é determinado. Esse valor é usado para marcar a posição relativa de uma afinidade no sistema.

Afinidades com Deltas positivos são impulsionadores relativos (relative drivers) ou direcionadores de gatilhos. As afinidades com Deltas negativos são efeitos relativos, resultados ou desfechos (relative effect or outcomes). Há o posicionamento de valor Delta como primeiro e segundo impulsionadores, primeiro e segundo 
desfechos e articuladores (circulator/pivot) quando há um mesmo número de INS e OUTS indicando uma posição da afinidade no meio do sistema. Esse é o elemento do qual depende todo o funcionamento do sistema. Há casos onde a Tabela IRD mostra todos os INS e OUTS com valores outros que zero. Nesses casos, isso indica que a afinidade é um forte efeito relativo, mas ainda é influenciado por ou influencia outras afinidades. Assim, aconselha-se nomear essas afinidades como primeiro impulsionador ou primeiro desfecho.

A Tabela 2 consolida cinco afinidades como impulsionadores (afinidade 1, 4, 5 e 7), quatro afinidades como desfecho (afinidades 2, 3, 6 e 9) e uma afinidade como pivô, a afinidade 8 . A contabilização das relações traz a métrica quantitativa para a análise qualitativa.

Tabela 2 Tabela IRD

\begin{tabular}{|c|c|c|c|c|c|c|c|c|c|c|c|c|}
\hline & 1 & 2 & 3 & 4 & 5 & 6 & 7 & 8 & 9 & OUT & IN & $\Delta$ \\
\hline 1 & & 4 & 4 & 4 & 4 & 4 & $\Lambda$ & 4 & $\uparrow$ & 6 & 2 & 4 \\
\hline 2 & 4 & & 4 & $\leftarrow$ & $\leftarrow$ & $\longleftarrow$ & 4 & 4 & 4 & 2 & 6 & -4 \\
\hline 3 & 4 & 4 & & 4 & 4 & 4 & 4 & 4 & 4 & 1 & 7 & -6 \\
\hline 4 & $\longleftarrow$ & 4 & 4 & & 4 & $\uparrow$ & 4 & 4 & 4 & 6 & 2 & 4 \\
\hline 5 & $\uparrow$ & $\uparrow$ & 4 & 4 & & $\uparrow$ & 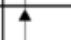 & 4 & 4 & 6 & 2 & 4 \\
\hline 6 & $\longleftarrow$ & 4 & 4 & $\leftarrow$ & 4 & & 4 & 4 & $\uparrow$ & 2 & 6 & -4 \\
\hline 7 & $\longleftarrow$ & $\uparrow$ & 4 & 4 & 4 & 4 & & 4 & $\uparrow$ & 5 & 3 & 2 \\
\hline 8 & 4 & 4 & 4 & 4 & 4 & $\longleftarrow$ & & & 4 & 4 & 4 & 0 \\
\hline 9 & $\longleftarrow$ & & 4 & 4 & & 4 & 4 & $\longleftarrow$ & & 3 & 5 & -2 \\
\hline
\end{tabular}

Fonte: Elaborado com base em NORTHCUTT, MCCOY, 2004, p.173.

Acrescentando a essa contabilidade, Northcutt e McCoy (2004) se utilizam do princípio de Pareto para determinar a relação de influência de poder de cada afinidade no sistema. O princípio de Pareto é um método estatístico que se baseia no princípio fundamental de que uma minoria das relações em qualquer sistema responderá por uma maioria da variação dentro do sistema. Northcutt e McCoy (2004) assumem que $20 \%$ das afinidades que serão classificadas de acordo com seu poder de influência, são responsáveis por $80 \%$ dos resultados causados por estas influências. A partir do protocolo do IQA, a planilha de Excel, Tabela 3 Protocolo de Pareto, foi preenchida. 
Tabela 3 Protocolo de Pareto

\begin{tabular}{|c|c|c|c|c|c|}
\hline $\begin{array}{l}\text { Rehação de par } \\
\text { de afinidade }\end{array}$ & $\begin{array}{l}\text { Frequência } \\
\text { Classificada } \\
\text { (Descendente) }\end{array}$ & $\begin{array}{l}\text { Frequência } \\
\text { Cumula tiva }\end{array}$ & $\begin{array}{c}\text { Percentual } \\
\text { Cumula tivo } \\
\text { (Relação) }\end{array}$ & $\begin{array}{l}\text { Percentual } \\
\text { cumula tivo } \\
\text { (frequência) }\end{array}$ & Poder \\
\hline $4>7$ & 6 & 6 & 1,4 & 2,5 & 1,1 \\
\hline $1>9$ & 6 & 12 & 2,8 & 4,9 & 2,1 \\
\hline $1>8$ & 6 & 18 & 4,2 & 7,4 & 3,2 \\
\hline $1>6$ & 6 & 24 & 5,6 & 9,8 & 4,3 \\
\hline $1>3$ & 6 & 30 & 6,9 & 12,3 & 5,4 \\
\hline $6>8$ & 5 & 35 & 8,3 & 14,3 & 6.0 \\
\hline $6>7$ & 5 & 40 & 9,7 & 16,4 & 6,7 \\
\hline $5>9$ & 5 & 45 & 11,1 & 18,4 & 7,3 \\
\hline $5>7$ & 5 & 50 & 12,5 & 20,5 & 8,0 \\
\hline $3>7$ & 5 & 55 & 13,9 & 22,5 & 8,7 \\
\hline $3<6$ & 5 & 60 & 15,3 & 24,6 & 9,3 \\
\hline $3<5$ & 5 & 65 & 16,7 & 26,6 & 10,0 \\
\hline $2>7$ & 5 & 70 & 18,1 & 28,7 & 10,6 \\
\hline $2>4$ & 5 & 75 & 19.4 & 30.7 & 11,3 \\
\hline $2>3$ & 5 & 80 & 20,8 & 32,8 & 12,0 \\
\hline $1>7$ & 5 & 85 & 22,2 & 34,8 & 12,6 \\
\hline $1>4$ & 5 & 90 & 23,6 & 36,9 & 13,3 \\
\hline $8>9$ & 4 & 94 & 25,0 & 38,5 & 13,5 \\
\hline $7>8$ & 4 & 98 & 26,4 & 40,2 & 13,8 \\
\hline $5>6$ & 4 & 102 & 27,8 & 41,8 & 14,0 \\
\hline $4>9$ & 4 & 106 & 29,2 & 43,4 & 14,3 \\
\hline $4>8$ & 4 & 110 & 30,6 & 45,1 & 14,5 \\
\hline $4<9$ & 4 & 114 & 31,9 & 46,7 & 14,8 \\
\hline $4<5$ & 4 & 118 & 33,3 & 48,4 & 15,0 \\
\hline $3>9$ & 4 & 122 & 34,7 & 50,0 & 15,3 \\
\hline $3>8$ & 4 & 126 & 36,1 & 51,6 & 15,5 \\
\hline $3>6$ & 4 & 130 & 37,5 & 53,3 & 15,8 \\
\hline $3>4$ & 4 & 134 & 38,9 & 54,9 & 16,0 \\
\hline $3 \leq 9$ & 4 & 138 & 48,3 & 56,6 & 16,3 \\
\hline 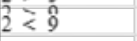 & 3 & 202 & $\begin{array}{l}117 \\
68,1\end{array}$ & 82,8 & 14,7 \\
\hline $2<8$ & 3 & 205 & 69,4 & 84,0 & 14,6 \\
\hline $2<6$ & 3 & 208 & 70,8 & 85,2 & 14,4 \\
\hline $2<4$ & 3 & 211 & 72,2 & 86,5 & 14,3 \\
\hline $1>2$ & 3 & 214 & 73,6 & 87,7 & 14,1 \\
\hline $1<2$ & 3 & 217 & 75,0 & 88,9 & 13,9 \\
\hline $6<7$ & 2 & 219 & 76,4 & 89,8 & 13,4 \\
\hline $5<9$ & 2 & 221 & 77,8 & 90,6 & 12,8 \\
\hline $5<8$ & 2 & 223 & 79,2 & 91,4 & 12,2 \\
\hline $5<6$ & 2 & 225 & 80,6 & 92,2 & 11,7 \\
\hline $4<8$ & 2 & 227 & 81,9 & 93,0 & 11,1 \\
\hline $3>5$ & 2 & 229 & 83,3 & 93,9 & 10,5 \\
\hline $3<4$ & 2 & 231 & 84,7 & 94,7 & 9,9 \\
\hline $2<7$ & 2 & 233 & 86,1 & 95,5 & 9,4 \\
\hline $2<3$ & 2 & 235 & 87,5 & 96,3 & 8,8 \\
\hline $1<7$ & 2 & 237 & 88,9 & 97,1 & 8,2 \\
\hline $7<8$ & 1 & 238 & 90,3 & 97,5 & 7,3 \\
\hline $5<7$ & 1 & 239 & 91,7 & 98,0 & 6,3 \\
\hline $4>5$ & 1 & 240 & 93,1 & 98,4 & 5,3 \\
\hline $1<9$ & 1 & 241 & 94,4 & 98,8 & 4,3 \\
\hline $1<6$ & 1 & 242 & 95,8 & 99,2 & 3,3 \\
\hline $1<4$ & 1 & 243 & 97,2 & 99,6 & 2,4 \\
\hline $1<3$ & 1 & 244 & 98,6 & 100,0 & 1,4 \\
\hline $1<8$ & 0 & 244 & 100,0 & 100,0 & 0,0 \\
\hline $\begin{array}{l}\text { Frequência } \\
\text { Total }\end{array}$ & 244 & $\begin{array}{r}\text { Ptequincie tota1 } \\
\text { igua1 }\end{array}$ & Ë zua 1 a $100 \%$ & Ė zeala $100 \%$ & Poder $=B-D$ \\
\hline
\end{tabular}

Fonte: Elaborado com base em NORTHCUTT, MCCOY (2004, p. 159). 
A coluna A descreve as possíveis relações entre as afinidades, a coluna $B$ descreve a frequência, em ordem decrescente de aparição de cada relação indicada individualmente pelos constituintes. A coluna $C$, por sua vez, traz a frequência cumulativa. As colunas $\mathrm{D}$ e $\mathrm{E}$ trazem o percentual que cada relação representa sobre o total de relações percebidas. $E$ a coluna $F$ indica o grau e poder de influência de cada relação. Seguindo o Princípio de Pareto, quando os valores estão preenchidos, é possível aferir que $20 \%$ das relações são representativas de $80 \%$ dos resultados, o que, segundo os autores, dá ao pesquisador segurança para descartar as demais relações e analisar mais profundamente apenas $20 \%$ delas.

A partir dessa planilha e do corte em $80 \%$, verificou-se o grau de consenso dos entrevistados sobre Diagrama de influência do Sistema, com o objetivo de entender quais eram as $20 \%$ das relações causais que afetavam os $80 \%$ dos resultados. Obteve-se 244 frequências das 72 possíveis relações feitas pelos constituintes. O número ideal de relações está no ponto em que o poder de influência no sistema atinge o máximo. O resultado do processo é a frequência de cada relação na ordem de afinidade. Uma avaliação da Tabela Pareto nesta pesquisa, indicou que, comparativamente, pouco mais de 48 relações possíveis foram responsáveis pela maior parte da variância, o que é consistente com o princípio de Pareto. O Gráfico 1 é uma ilustração da variância contabilizada da frequência acumulada em relação à quantidade de relações entre pares de afinidade da pesquisa que ilustra esse artigo.

\section{Gráfico 1 Maximização da Variância}

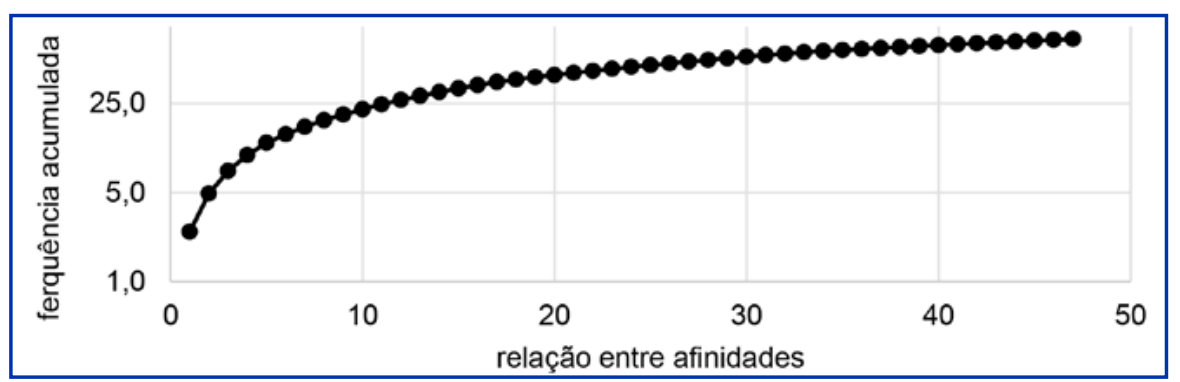

Fonte: Elaborado pelos autores (2021).

O Gráfico 2 mostra que o ápice de poder de influência é conseguido no índice de 34 relações de afinidades que representa $61,1 \%$ da variação neste sistema. Essa 
é a relação da afinidade 1 com a afinidade 5, em que o Ecossistema(afinidade 5) é a causa que impulsiona a Liderança com Propósito(afinidade 1).

\section{Gráfico 2 Poder de influência}

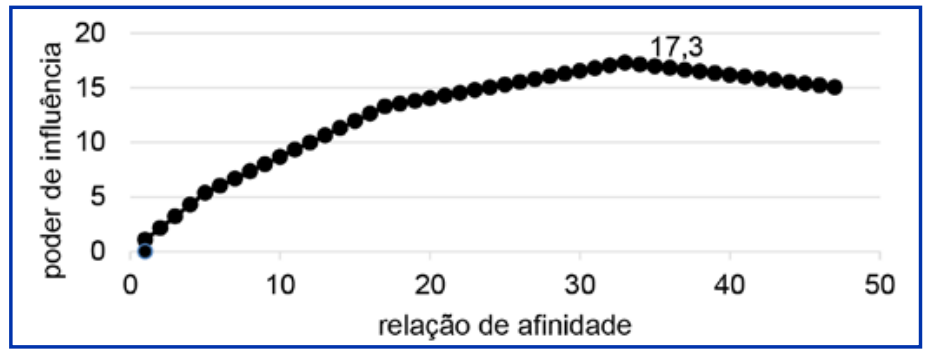

Fonte: Elaborado pelos autores (2021).

Ao decidir quais relações incluir ou excluir do Diagrama composto (IRD), as duas últimas colunas da Tabela Pareto são fundamentais para determinar o ponto de corte à medida que as relações são exibidas em ordem de frequência decrescente. As relações 49 a 72 foram excluídas. Foram feitos dois IRD compostos. O primeiro apenas considerando as relações de causa ou efeito e pela maior frequência e poder de influência. Foram obtidas apenas quatro possíveis relações e com as posições no sistema conforme descrito na Figura 3. O Ecossistema é a única afinidade de consenso que tem apenas uma direção na relação com outras afinidades. O Ecossistema é a causa que influencia os Resultados de Negócio e os Resultados Sociais e é influenciada pelas afinidades Desenvolvimento Econômico Sustentável e Impacto.

Figura 3 Topologia de afinidades de consenso

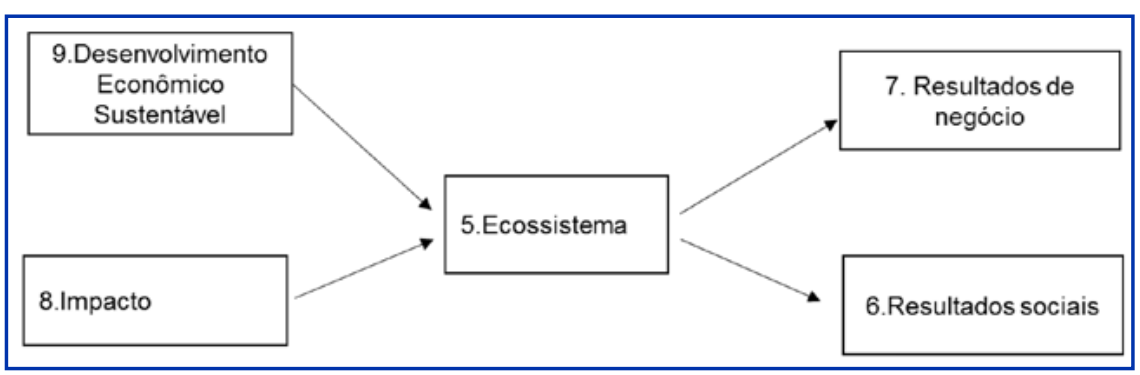

Fonte: Elaborado pelos autores (2021). 
Para essa seleção de relações chegamos ao Diagrama de Influência do Sistema Composto (SID composto), em que o Desenvolvimento Econômico Sustentável e Impacto são as causas impulsionadoras do sistema e Resultados Sociais e de Negócios são os desfechos. Ecossistema é a afinidade articuladora do sistema (Figura 3). A seleção das relações considerando as conflitantes, isto é, as que indicam causa e efeito ao mesmo tempo e com maior frequência configurou um outro Diagrama. No SID composto, a Liderança com Propósito é a causa impulsionadora de todo o sistema sendo influenciada apenas pelo Ecossistema. E o Resultado de Negócios é o único primeiro desfecho, aquele que não influencia nenhuma outra afinidade, como demonstrado na Figura 4.

Figura 4 SID Composto

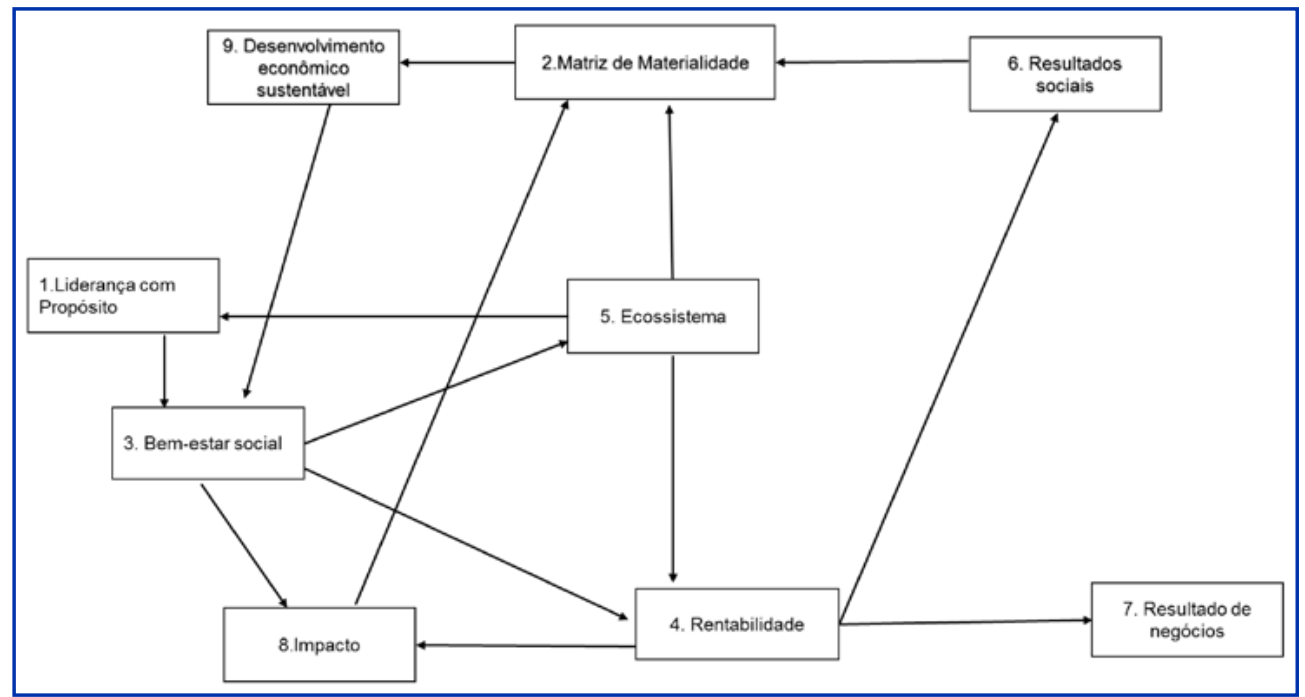

Fonte: Elaborado pelos autores (2021).

O Diagrama do Sistema demonstra o caminho no processo da adoção da Criação do Valor Compartilhado. Uma possível análise de resultados é que a afinidade impulsionadora de todo o sistema é a Liderança com Propósito que, a partir do objetivo estratégico de gerar Bem-estar Social e Impacto, influencia a definição da Matriz de Materialidade que, por sua vez, influencia todo o Desenvolvimento Econômico Sustentável da organização. Uma outra análise possível é que o Desenvolvimento 
Econômico Sustentável volta a influenciar a geração de bem-estar social que impacta o Ecossistema, devolvendo feedbacks (loops) e alimentando a Liderança com Propósito e a constante análise da Matriz de Materialidade. Essa alimentação constante vem dos retornos de bem-estar social e Impacto que por sua vez são oriundos do Ecossistema. O Ecossistema está posicionado no meio fazendo todo o Sistema funcionar. Esta afinidade influencia diretamente a Liderança com Propósito, a Matriz de Materialidade e a Rentabilidade e influencia indiretamente todas as outras afinidades. É, portanto, dessa forma, que o pesquisador vai desenvolvendo a análise das relações entre os elementos e validando sua leitura com os participantes (constituintes).

Os constituintes elaboram um SID desordenado (cluttered), que é revisado e validado na construção conjunta com o pesquisador, eliminando-se as relações duplicadas e obtendo-se um SID limpo (clear). Por fim, junta-se ao SID limpo às significações ou citações teóricas fornecidas pelos constituintes na Tabela de relações entre afinidades (ART). A Figura 5, traz o SID finalizado de uma das empresas da amostra da pesquisa que exemplificou esse artigo.

Figura 5 SID finalizado

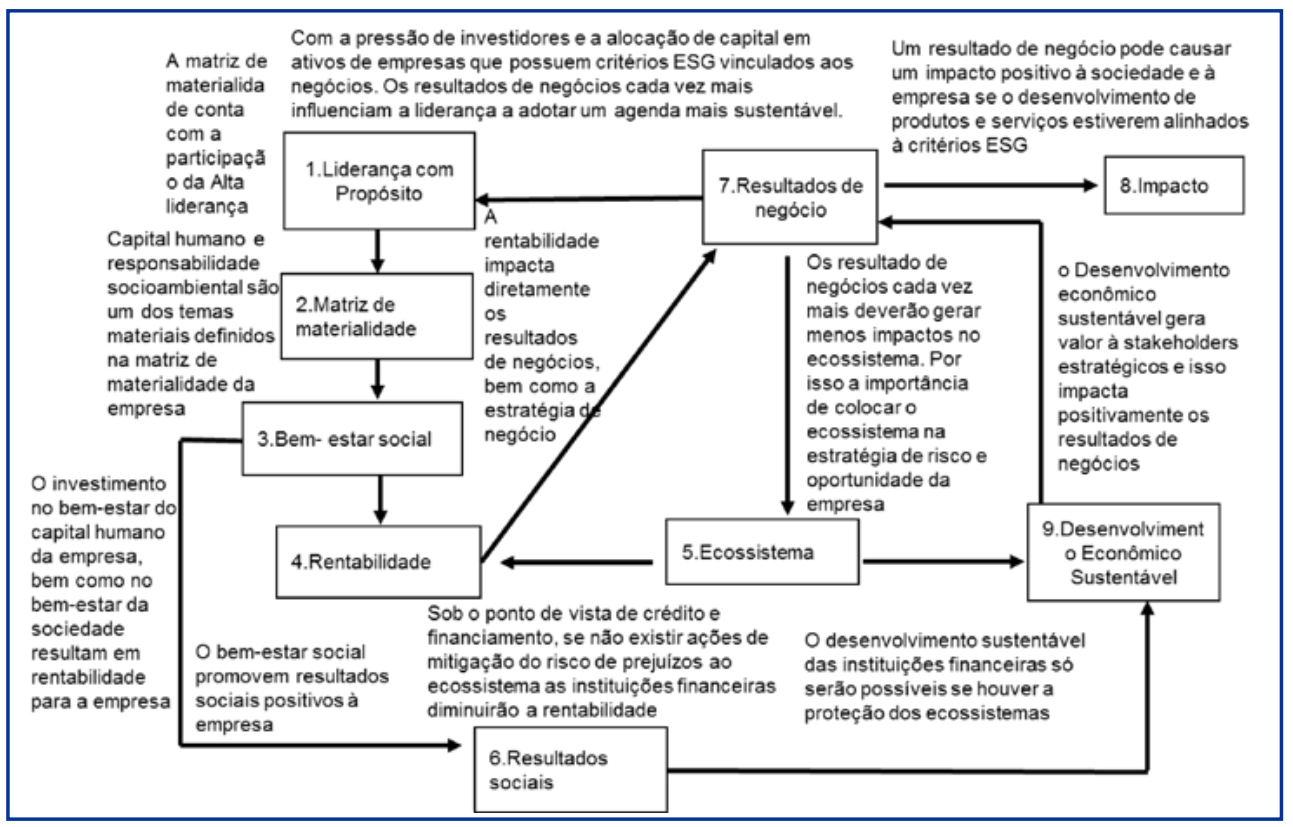

Fonte: Elaborado pelos autores (2021). 
Os resultados obtidos nesse processo fornecem subsidios para o protocolo de entrevistas individuais. Nessa etapa, a experiência coletiva será investigada na perspectiva individual.

De acordo com o método IQA, as entrevistas semiestruturadas começam com uma conversa entre o pesquisador e o entrevistado, a partir do SID (Diagrama de influência de sistema). Desta forma, o pesquisador não induz as respostas e objetiva captar a percepção individual dos resultados obtidos na coletividade.

Northcut e McCoy definem que "O papel do pesquisador passa de designer para facilitador, ensinando aos membros do grupo o processo e orientando-os a gerar e analisar seus próprios dados com influência externa mínima." (NORTHCUTT; MCCOY, 2004, p. 44). Consequentemente, os vieses e preconceitos do pesquisador são minimizados no processo.

O método IQA ressalta que um sistema social e seus elementos envolvem a interpretação humana de significados; isto é, o que faz as coisas acontecerem e por quê. Sendo assim, sete codificações teóricas foram desenvolvidas fazendo uma "colcha de retalhos" com as transcrições das entrevistas.

\section{Discussão do Método IQA}

O método IQA apresenta uma consistente técnica de análise de dados por meio da combinação de duas técnicas de coleta de dados qualitativos - entrevista e grupo focal - de forma a valorizar o melhor de cada uma dessas ferramentas. Pela combinação dessa técnica e a análise de dados conjunta entre pesquisador e participantes, IQA busca minimizar a interferência no viés do pesquisador nos resultados da pesquisa. Entretanto, o método não propõe um protocolo para minimizar o viés dos participantes.

Um dos pontos fortes do método é centrado no conjunto de protocolos que o pesquisador utiliza para gerar as diferentes planilhas que transformam a percepção qualitativa dos participantes em códigos numéricos. Dessa forma, reduzindo possíveis interferências na análise dos dados por parte do pesquisador e focando na percepção daqueles que de fato conhecem e atuam no fenômeno estudado. 
$\mathrm{Na}$ pesquisa foi possível identificar elementos considerados muitas vezes contraditórios nas percepções dos participantes por meio da correlação entre as variáveis Rentabilidade e Bem-estar Social. Por ser uma análise qualitativa e com uma lógica binária na relação entre um par de afinidades, foi identificada a correlação positiva entre essas duas variáveis nos depoimentos dos entrevistados.

O método IQA propõe três possíveis relações entre um par de afinidades: causa, efeito ou nenhuma. É importante destacar que no estudo cerca de $50 \%$ dos constituintes identificaram uma quarta relação de causa e efeito em um par de afinidades. A relação onde um par de afinidade é ao mesmo tempo causa e efeito, indicado no protocolo ART por seta de dois sentidos $(<>)$. Essa nova relação permite a coexistência de dois valores de verdade.

Há indício empíricos de uma mudança de percepção de gestores empresariais que buscam a conciliação entre objetivos antes excludentes e contraditórios, como, por exemplo, a visão de ser impossível conciliar rentabilidade e bem-estar social. Nesse sentido, é evidenciada a mudança da lógica clássica do pensamento linear binário do "ou isso ou aquilo" para a lógica do pensamento complexo de Morin, admitindo o conectivo "e". Assim como colocado por Morin (2011), o pensamento complexo é uma teoria que aceita e procura compreender as muitas faces e as mudanças constantes do real e não pretende negar a multiplicidade, a aleatoriedade e a incerteza, em que a contradição pode conviver. Nesse sentido, essa mudança de paradigma de pensamento, contribui para o entendimento das problemáticas empresariais complexas da área de sustentabilidade.

O método reforça a ideia de que não conhecemos a realidade como ela é, mas sim uma imagem representativa do momento. Os protocolos do IQA materializam a percepção em diagramas ou mapa mentais elaborados coletivamente por aqueles envolvidos no fenômeno em estudo. Em outros termos, cada constituinte formaliza seu mapa mental de acordo com o modo como se preparou para assumir a elaboração do sistema através de seus elementos e suas relações.

O fenômeno social pode, assim, mostrar aspectos contraditórios que seriam incompreensíveis do ponto de vista da lógica clássica. O modelo mental linear pode ser necessário para lidar com os problemas das ciências exatas, mas não é suficiente para resolver problemas humanos em que participem emoções e sentimentos (a dimensão social). Essa mudança no paradigma da "lógica clássica" para o "pensamento complexo" está expressa na significação de cada afinidade desenvol- 
vida e validada pelos constituintes. A metodologia não considera a possibilidade da relação simétrica de causa e efeito entre um par de afinidades. Nesse sentido, poderia ser considerada a utilização dos diferentes conectivos existentes entre proposições lógicas compostas. Os constituintes da pesquisa ora relatada entenderam, por exemplo, que há a possibilidade de relacionar A e B além do conectivo "ou", que considera que apenas uma proposição é verdadeira. O conectivo "e" considera possível ter duas proposições verdadeiras ao mesmo tempo.

\section{Coniderações Finais}

Esse artigo propôs apresentar uma opção metodológica para pesquisas complexas de sustentabilidade. Nesse sentido, o método IQA de Northcut e McCoy (2004) traz contribuições importantes para a abordagem qualitativa à medida que incorpora aspectos da abordagem quantitativa.

Os protocolos baseados em tabelas e planilhas que utilizam números trazem rigor a pesquisa e minimizam o viés do pesquisador. Os significados desses números aparecem na fase final de comparação dos diagramas. A validação dos diagramas coletivos pelas entrevistas individuais contribui para minimizar vieses dos participantes e do pesquisador. Ademais, os protocolos permitem que outros pesquisadores os apliquem para investigar o mesmo fenômeno em outros contextos. Ou ainda, que em um mesmo contexto, envolva outros constituintes. A análise comparativa de acordo com os protocolos e procedimentos do método, pode contribuir para o entendimento de fenômeno cuja literatura ainda está em construção.

O método IQA pressupõe que os participantes estejam ativamente engajados na coleta e análise dos dados, nesse sentido, ele pode ser considerado um método inovador para a discussão de temas na área de sustentabilidade. Entretanto, a possibilidade de alteração dos diagramas através da validação dos participantes pode trazer vieses aos resultados. O método peca em não oferecer um protocolo para esse ponto.

Desta forma, recomenda-se, que mais pesquisas com abordagem qualitativa e que procurem respostas para questões compelxas de diferentes áreas adotem o método IQA. 


\section{Referências Bibliográficas}

BARGATE, K. Interactive Qualitative Analysis - A novel methodology for qualitative research. Mediterranean Journal of Social Science, vol. 5, no. 20, pp. 11-19, 2014. https://www.mcser.org/journal/index.php/ mjss/article/download/3704/3629.

BARNEY, J. B. Firm resources and sustained competitive advantage. Journal of Management, v. 17, n. 1, p. 99- 120, 1991.

BISHWAKARMA, G. Nepalese Schoolchildren as Research Participants: Challenges in Qualitative Research. Open Journal of Social Sciences, 5, 52-68. 2017. http://dx.doi.org/10.4236/jss.2017.51005.

DENZIN, N. K.; LINCOLN, Y. S. Introduction: the discipline and practice of qualitative research. The Sage Handbook of qualitative research. 4. ed. Thousand Oaks: Sage, 2000. p. 1-32.

GIL, A. C. Métodos e técnicas de pesquisa social. 4 ed. São Paulo: Atlas, 1995.

GLASER, B.; STRAUSS, A. The discovery of grounded theory. New York: Aldene de Gruyter, 1967. 271p. KRISHNAN, G.L.; PRAKASH, S. Adoption of Health and Fitness Apps by Smartphone Users: Interactive Qualitative Analysis. 10.23919/PICMET.2019.8893855.

KUHN, T. S. A estrutura das revoluções científicas. Tradução :Beatriz Vianna Boeira e Nelson Boeira. - 12. Ed. São Paulo: Perspectiva, 2013.

LESSA, B.; SPIER; NASCIMENTO, L.F.M. do. As Barreiras para a Sustentabilidade em Escolas de Administração: uma explicação Bourdieusiana. Administração: Ensino E Pesquisa, 19(3), 555-582. https://doi. org/10.13058/raep.2018.v19n3.955

LUKOSEVICIUS, A. P. Executar é preciso, planejar não é preciso: proposta de framework para projetos de pesquisa. Administração: Ensino e Pesquisa, 19(1), 32-65. https://doi.org/10.13058/raep.2018.v19n1.765 MORIN, E. Introdução ao pensamento complexo. Tradução: Eliane Lisboa. Porto Alegre: Sulina. 2011. MORIN, E. Os sete saberes necessários à educação do futuro. Tradução: Catarina Eleonora F. da Silva e Jeanne Sawaya. Revisão técnica de Edgard de Assis carvalho.2. ed. rev. São Paulo. Cortez; Brasília, DF. UNESCO, 2011.

NIELSEN et al. Guia Prático Para Elaboração de Monografias, Dissertações e Teses em Administração. São Paulo: Saraiva Educação, 2018.

NORTHCUTT, N.; MCCOY, D. Interactive qualitative analysis: A systems method for qualitative research. Sage, London, 2004.

PORTER, M. E.; KRAMER, M. R. Creating shared value: how to reinvent capitalism and unleash a wave of innovation and growth. Harvard Business Review 89(1/2), 2011, pp. 62-77.

PORTER, M. E.; KRAMER, M. R. Strategy and Society: The Link Between Competitive Advantage and Corporate Social Responsibility. Harvard Business Review. December 2006.

PREEZ, H. Taxation students' perceptions of open-book assessment: a follow-up interactive qualitative analysis. South African Journal of Accounting Research. 29. 84-99. 10.1080/10291954.2015.999457.

PREEZ, H.; STIGLINGH, M. Confirming the fundamental principles of taxation using Interactive Qualitative Analysis, e-Journal of Tax Research (2018) vol. 16, no. 1, pp. 139-174.

PUGALIA, SANCHEETA; SAI, L. Student Entrepreneurs' Perspective on the Role of Personal Networks in 
Venture Creation: An Interactive Qualitative Analysis. Conference: Australian Centre for Entrepreneurship Research Exchange. At: Gold Coast, Australia. Feb,2016.

SANCHEZ, J. Second life: An interactive qualitative analysis. Proceedings of Society for Information Technology and Teacher Education International Conference 2007. 1240-1243.

SANTOS, V.; BACCI, D. Proposta para governança ambiental ante os dilemas socioambientais urbanos. Estudos Avançados. São Paulo, v. 31, n. 89, p. 199-212, Apr. 2017. Available from <http://www.scielo.br/scielo.php?script= sci_arttext\&pid=S0103-40142017000100199\&lng=en\&nrm=iso $>$. access on 08 Oct. 2020. https://doi.org/10.1590/s0103-40142017.31890017.

SOUZA, M.; RIBEIRO, H. Sustentabilidade Ambiental: uma Meta-análise da Produção Brasileira em Periódicos de Administração. Revista de Administração Contemporânea (RAC), Rio de Janeiro, v. 17, n. 3, art. 6, pp. 368-396, maio/jun. 2013.

STRAUSS, A.; CORBIN, J. Basics of qualitative research. Thousand Lage Daks: Lage Publications, 1990. $267 \mathrm{p}$.

ZAPPELLINI, M. B.; FEUERSCHÜTTE, S. G. O uso da triangulação na pesquisa científica brasileira em administração. Administração: Ensino e Pesquisa, 16(2), 241-273. https://doi.org/10.13058/raep.2015.

v16n2.238 